\title{
Contrast Transfer and Resolution Limits for Sub-Ångström High-Resolution Transmission Electron Microscopy
}

\section{Lentzen and Knut Urban}

Institute of Solid State Research, Ernst Ruska Centre for Microscopy and Spectroscopy with Electrons, Research Centre Jülich, 52425 Jülich, Germany

In the past years the information limit of high-resolution transmission electron microscopes has improved notably through the combined use of new instrumentation, such as field-emission sources, more stable lens and high-voltage power supplies, spherical-aberration correctors, and, recently, gun monochromators. The interpretability of high-resolution images has improved particularly due to the strong reduction of contrast delocalisation in the image plane by spherical-aberrration correction. At the same time exit-wave reconstruction, either by focus-variation or electron holography, in tandem with numerical aberration-correction has further improved the interpretable resolution of structure images.

Today high-resolution electron microscopy is about to penetrate the sub-Ångström scale considerably, and it is therefore important to explore how far that improvement will further the interpretable resolution. The high-energy scattering of electrons by the projected potential of a thin specimen generates a local phase modulation of the exit wave function, and the subsequent imaging by electron optic lenses forms the image intensity, which is recorded by a camera. It is therefore instructive to assess and compare the interpretable resolution at three levels: the projected potential map, the phase map of the exit wave function, and the recorded image intensity.

For many materials science investigations a direct view of the projected atomic structure and its assessment by an observer is the starting point for modelling the object of interest. We have adopted in this work the common Rayleigh criterion in order to evaluate the interpretability of a structure image. If the contrast peaks of two adjacent atomic columns are considered, the criterion states that the pair of columns appears resolved if the distance between the two peaks is larger than $\sim 1.15$ times the full width at half maximum (FWHM) of the peaks. The pair of columns appears just resolved if the contrast between the columns drops to $\sim 0.81$ times the peak contrast.

For this simulation study on interpretable resolution we have chosen hypothetical model structures of isolated $\mathrm{C}, \mathrm{Si}$, and Ge columns each comprising 5 atoms with an inter-atom spacing of $0.4 \mathrm{~nm}$ along the columns. Using the EMS software package [1] the respective projected potential maps, phase maps of the exit wave function, and image intensities were calculated for a hypothetical 300$\mathrm{kV}$ microscope with an information limit of $0.05 \mathrm{~nm}$. The microscope was set to the bright-atom contrast condition [2,3], at an overfocus of $2.2 \mathrm{~nm}$ and a negative third-order spherical aberration of $-1.85 \mu \mathrm{m}$, where the Scherzer pass-band extends to the information limit. In order to compare the effect of different scattering powers of $\mathrm{C}, \mathrm{Si}$, and $\mathrm{Ge}$, the simulation conditions for electron scattering were otherwise equal; in particular a Debye-Waller factor of $0.006 \mathrm{~nm}^{2}$ was chosen throughout. The contrast width of the $\mathrm{C}, \mathrm{Si}$, and Ge columns was measured by drawing line-scans across the peaks in the potential maps, the phase maps of the exit wave function, and the image intensity maps. The results are compiled in Table 1. Then the minimum distance at which two adjacent columns should appear resolved to an observer were calculated via the Rayleigh criterion. The resolved distances are compiled in Table 2.

From the simulated contrast widths the following tendencies can be concluded: 1 . In the potential map the weakly scattering $\mathrm{C}$ column appears wider than the Si and Ge columns. 2. In the phase map of the exit wave function the apparent column width increases with decreasing scattering power. 3. In the intensity map the strongly scattering Ge column appears narrower than the $\mathrm{C}$ and $\mathrm{Si}$ columns, and together with the peaks sidelobes appear. 4. The contrast width is larger in the phase map than in 
the potential map. 5. The contrast width is narrower in the intensity map than in the phase map. Observations 2 and 4 are in accordance with the quantum-mechanical properties of the first bound state of a potential, in the framework of the channelling theory of electron scattering. Observation 5 is at first sight suprising, because one might expect that the additional point-spread of the microscope should increase the contrast width compared to the exit wave. The image intensity, however, is the square of the exit wave convoluted with the point-spread function. Taking the square "sharpens" the peaks, and this effect over-compensates the blur induced by the small point-spread of a corrected instrument.

From the resolved distances, which were calculated from the contrast widths via the Rayleigh criterion, additional tendencies can be concluded: 1 . The hypothetical microscope has an information limit of $0.05 \mathrm{~nm}$, yet only a $\mathrm{Ge}-\mathrm{Ge}$ column distance of $0.05 \mathrm{~nm}$ can be resolved; $\mathrm{Si}-\mathrm{Si}$ and $\mathrm{C}-\mathrm{C}$ column distances smaller than $0.07 \mathrm{~nm}$ are not resolved. 2. The resolved distance, and hence the interpretable resolution, is dependent on the scattering power of the column pair. 3 . The interpretable resolution in the potential map poses a limit of $0.045 \mathrm{~nm}$ for any direct structural view.

Values for the resolved distance in the image intensity map, calculated as before, may be falsified by the above-mentioned appearance of sidelobes. In order to evaluate their effect, maps for two Si column pairs were simulated, with inter-column distances of $0.07 \mathrm{~nm}$ and $0.08 \mathrm{~nm}$, and all other parameters as above. Both column pairs are well-resolved in the potential map; in the phase map of the exit wave function the $0.08-\mathrm{nm}$ pair is well-resolved, the $0.07-\mathrm{nm}$ pair just resolved; in the image intensity map the $0.08-\mathrm{nm}$ pair is just resolved, see Figure 1 (left), the $0.07-\mathrm{nm}$ pair is not resolved, see Figure 1 (right). Hence, the sidelobes induce in the image intensity map an additional loss of interpretable resolution of about $0.01 \mathrm{~nm}$. The respective corrected values are shown in Table 2 , last column: the interpretable resolution of the intensity map ranges from $0.06 \mathrm{~nm}$ to $0.08 \mathrm{~nm}$, dependent on the scattering power and despite an instrumental information limit of $0.05 \mathrm{~nm}$.

\section{References}

[1] P. Stadelmann, Ultramicroscopy 21 (1987) 131.

[2] C.L. Jia, M. Lentzen, K. Urban, Microsc. Microanal. 10 (2004) 174.

[3] M. Lentzen, Ultramicroscopy 99 (2004) 211.

Table 1. Simulated contrast widths (FWHM) of atom columns

\begin{tabular}{llll}
\hline Atom & Potential $(\mathrm{nm})$ & Exit wave $(\mathrm{nm})$ & Image intensity $(\mathrm{nm})$ \\
\hline $\mathrm{C}$ & 0.047 & 0.070 & 0.059 \\
$\mathrm{Si}$ & 0.039 & 0.063 & 0.059 \\
$\mathrm{Ge}$ & 0.039 & 0.047 & 0.044 \\
\hline
\end{tabular}

Table 2. Interpretable resolution of atom column distances

\begin{tabular}{lllll}
\hline Atom & Potential $(\mathrm{nm})$ & Exit wave $(\mathrm{nm})$ & Image intensity $(\mathrm{nm})$ & Image intensity/sidelobes $(\mathrm{nm})$ \\
\hline $\mathrm{C}$ & 0.054 & 0.081 & 0.068 & 0.078 \\
$\mathrm{Si}$ & 0.045 & 0.072 & 0.068 & 0.078 \\
$\mathrm{Ge}$ & 0.045 & 0.054 & 0.051 & 0.061 \\
\hline
\end{tabular}
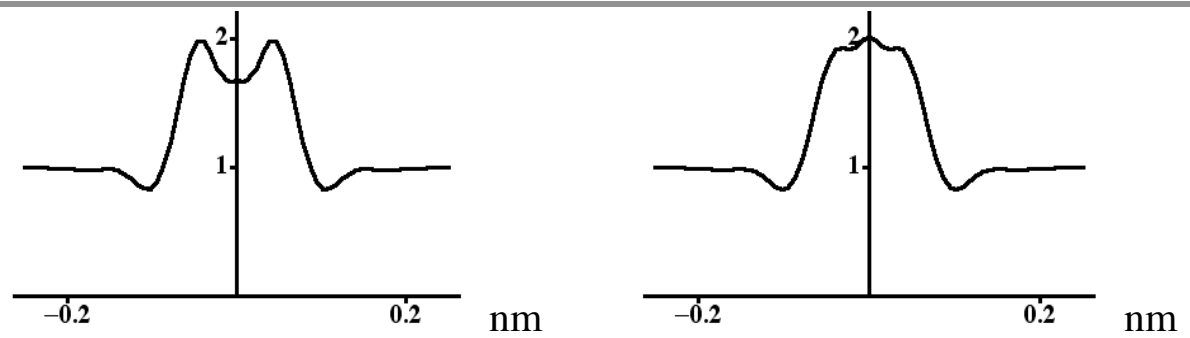

Fig. 1. Image intensity across a pair of Si columns. Column distance: (left) $0.08 \mathrm{~nm}$, (right) $0.07 \mathrm{~nm}$. 